\title{
Hung's Difficult and Failed Airway Management, Third Edition
}

\author{
Orlando Hung, Michael F. Murphy. McGraw Hill Education, 2018, 695 pages, \\ ISBN 978-1-259-64054-4
}

\author{
Andrea Petropolis, MD
}

Received: 12 July 2018/Accepted: 12 July 2018/Published online: 3 October 2018

(c) Canadian Anesthesiologists' Society 2018

In the third edition of this textbook, Hung and Murphy provide a comprehensive and up-to-date review of difficult and failed airway management. The book is clinically focused and is a pleasure to read. Although the overall content is similar to that in previous editions, a number of additional chapters cover new topics. A broad range of airway devices are discussed, and this new edition contains both updated references and airway management algorithms.

The first section, Principles of Airway Management, comprises nine chapters covering the foundations of airway management. Airway evaluation, pharmacology, and algorithms are discussed, and extensive references are provided. This section contains an excellent chapter dedicated to preparation for awake intubation, including a highly detailed and well-illustrated discussion of airway anatomy and local anesthetic topicalization. Another interesting chapter focuses on aspiration risks and preventive measures, including up-to-date fasting guidelines and the use of cricoid pressure. It is refreshing to revisit basic airway topics from an evidence-based perspective and to learn what the literature says about dayto-day anesthesia practices.

The second section, Airway Techniques, contains nine chapters and provides an overview of the many tools and techniques that may be used in airway management, including various laryngoscopes, video laryngoscopes, bronchoscopes, and extraglottic airway devices. All are described in great detail from historical, technical, and clinical perspectives. Although far more tools and techniques are described than most anesthesiologists will ever use, this section nonetheless serves as a useful

\footnotetext{
A. Petropolis, MD $(\bowtie)$

Department of Anesthesiology, Perioperative and Pain Medicine, University of Manitoba, Winnipeg, MB, Canada

e-mail: apetropolis@me.com
}

reference and perhaps a starting point for expanding one's repertoire. Basics are also discussed, including bagmask ventilation and direct laryngoscopy. Once again, it is interesting to reexamine these more conventional techniques through an evidence-based lens. There is also an excellent chapter covering cricothyrotomy, with many illustrations and a clear description of how to execute this rarely performed but exceedingly important procedure.

The third to ninth sections pertain to airway management in specific settings, including Pre-Hospital Airway Management, Airway Management in the Emergency Room, Airway Management in the ICU, Airway Management in the Operating Room, Airway Management in the Pediatric Population, Airway Management in Obstetrics, and Airway Management in Unique Environments. Each chapter in these sections follows a case-based discussion format, beginning with a clinical vignette, followed by a discussion of relevant airway considerations, including patient assessment, management options, and any special considerations relating specifically to the situation in question. The clinical vignettes are numerous and diverse. Some describe situations that many of us are unlikely ever to encounter, such as intubating an unconscious patient trapped inside a vehicle or airway management under combat conditions. Although applicability may be limited, it is nonetheless interesting to learn how to address these challenging airway situations. Most of the vignettes, however, describe scenarios that have broad applicability. For instance, airway management in the setting of deep neck infection, penetrating neck trauma, or expanding neck hematoma are all topics of relevance to any anesthesiologist. The wide range of themes discussed provides an excellent overview of airway management in a variety of difficult situations. As in the rest of the book, these chapters are well referenced and written in a clinically relevant and readable tone. Information is 
organized in an easy-to-follow question-and-answer format, which allows the reader to easily tap into the broad clinical experience of the authors and editors.

The final section of the text is entitled Practical Considerations in Airway Management. This section includes chapters on difficult airway carts, documentation of difficult and failed airway management, and teaching and simulation of airway management.

The content of this book can also be accessed online through Access Anesthesiology (https://accessanesthesiology. mhmedical.com/book.aspx?bookid=2206). In addition to the entire text being available online, further content includes several online narrated videos demonstrating airway and other related techniques (http://DifficultAirwayVideos.com). These videos are a useful supplement to the text and can help a reader quickly understand how to use an unfamiliar airway device. There are also several videos demonstrating basic techniques, such as direct laryngoscopy, which would serve as a useful teaching tool when working with junior trainees.
In conclusion, Hung's Difficult and Failed Airway Management, Third Edition provides an excellent overview of difficult and failed airway management, a topic of fundamental importance to the specialty of anesthesiology. The content is evidence-based and clinically focused. This book would be a useful addition to the library of anesthesia trainees as they learn to manage complex airways or prepare for board examinations. The Third Edition also serves as a useful reference text for practicing anesthesiologists interested in refreshing and updating their knowledge, or those seeking to learn about newer airway devices and techniques.

Conflicts of interest None declared.

Editorial responsibility This submission was handled by Dr. Hilary P. Grocott, Editor-in-Chief, Canadian Journal of Anesthesia. 